\title{
Collapse of an initially spherical vapour cavity in the neighbourhood of a solid boundary
}

\author{
By MILTON S. PLESSET AND RICHARD B. CHAPMAN \\ California Institute of Technology
}

(Received 5 June 1970 and in revised form 30 October 1970)

Vapour bubble collapse problems lacking spherical symmetry are solved here using a numerical method designed especially for these problems. Viscosity and compressibility in the liquid are neglected. Two specific cases of initially spherical bubbles collapsing near a plane solid wall were simulated: a bubble initially in contact with the wall, and a bubble initially half its radius from the wall at the closest point. It is shown that the bubble develops a jet directed towards the wall rather early in the collapse history. Free surface shapes and velocities are presented at various stages in the collapse. Velocities are scaled like $(\Delta p / \rho)^{\frac{1}{2}}$ where $\rho$ is the density of the liquid and $\Delta p$ is the constant difference between the ambient liquid pressure and the pressure in the cavity. For

$$
\Delta p / \rho=10^{6} \mathrm{~cm}^{2} / \mathrm{sec}^{2} \approx 1 \text { atm } / \text { density of water }
$$

the jet had a speed of about $130 \mathrm{~m} / \mathrm{sec}$ in the first case and $170 \mathrm{~m} / \mathrm{sec}$ in the second when it struck the opposite side of the bubble. Such jet velocities are of a magnitude which can explain cavitation damage. The jet develops so early in the bubble collapse history that compressibility effects in the liquid and the vapour are not important.

\section{Introduction}

The study of the behaviour of a bubble in a liquid is greatly simplified by the assumption of spherical symmetry. Following Rayleigh's (1917) classical analysis of a problem first solved by Besant, the inviscid collapse of a spherical cavity in a homogeneous, incompressible liquid under a constant ambient pressure, numerous authors have studied the behavour of spherical bubbles under a wide range of conditions. Far less is known about the non-spherical behaviour of bubbles. Because problems lacking spherical symmetry have proved too complex for direct analysis, they have been investigated primarily by qualitative reasoning, experiments, and perturbations from spherically symmetric solutions.

A problem of primary importance is the interaction of a collapsing bubble with a solid surface. The earliest theory of cavitation damage was based on the high pressures developed near a spherical cavity which has collapsed to a small fraction of its initial size. A more recent theory includes the pressures developed during rebound caused by the compression of a small amount of permanent gas contained in the bubble. Calculations discussed by Plesset (1966) indicate that stresses produced by the collapse and subsequent rebound of a spherical bubble 
fall off rapidly as the distance from the bubble is increased and are too small to damage a solid surface unless the surface is quite close to the bubble. Thus the presence of a solid boundary will have an important effect in destroying the spherical symmetry of any bubble capable of producing damage.

Another explanation of cavitation damage is the theory, first suggested by Kornfeld \& Suvorov (1944), that damage is caused by the action of liquid jets formed on bubbles near the solid surface. A perturbation study by Rattray (1951) suggested that the effect of a solid wall in disturbing the flow during the collapse of an initially spherical bubble could cause the formation of a liquid jet directed towards the wall. Experiments by Benjamin \& Ellis (1966) later confirmed that jets form on bubbles collapsing near a solid wall. Large vapour bubbles, generally about one centimetre in radius, were grown from small nuclei by the application of a negative pressure. High-speed photographs were taken of these bubbles as they collapsed near a plane solid surface. The ambient pressure was maintained at about 0.04 atm during collapse so that collapse velocities would be reduced to facilitate the photography. These bubbles were nearly spherical as they started collapsing. First, they became elongated in the direction normal to the wall; then they tended to flatten and form an inward moving jet on the side of the bubble opposite the wall.

The advantages of a numerical technique for stimulating non-spherical bubble collapse are clear. Experiments are difficult and give only sketchy results. Perturbations from spherically symmetric solutions are not valid for large deformations. A numerical solution, however, can check results and supply detailed information. Numerical methods can also be applied to situations which might be very difficult to produce in the laboratory. Mitchell, Kling, Cheesewright \& Hammitt (1967) have considered simulation of bubble collapse using the Markerand-Cell technique, a general method for simulating incompressible, viscid flows with an assortment of boundary conditions including free surfaces. Because non-spherical collapse is of such interest, it is worthwhile to develop a method of simulation especially suited to these problems.

\section{Definition of the problem}

The asymmetries caused by a solid wall should be separated from those due to initial asymmetries in shape or velocity of the type analyzed in the linearized theory of Plesset \& Mitchell (1956). The bubble is therefore taken to be spherical and at rest at the initiation of the collapse, and any other extraneous asymmetric effects such as gravity are also omitted.

The following assumptions will be made: (1) The liquid is incompressible. (2) The flow is non-viscous. (3) The vapour pressure is uniform throughout the bubble interior. (4) The ambient pressure and the vapour pressure are constant with time. (5) The bubble contains no permanent gas. (6) Surface tension effects are negligible.

This set of assumptions defines the problem as the non-spherical version of the classical Rayleigh collapse calculation. Only the first three assumptions are essential to the method of simulation developed here. The last three assumptions 
are made to keep the essential features of the problem in the foreground. With the absence of shocks, compressibility will not become important until speeds in the liquid are comparable with the speed of sound. Thus the liquid can be assumed to be incompressible with the understanding that solutions are valid for small Mach numbers only. In most cases of collapse, viscosity can be neglected unless the bubble is initially very small. For example, viscosity is unimportant for a spherical bubble collapsing in water under atmospheric pressure if the initial radius is $10^{-3} \mathrm{~cm}$ or greater. As for the assumption of uniform pressure inside the bubble, this assumption will remain valid as long as speeds on the bubble surface are below the speed of sound in the vapour.

The problem is specified by the following conditions: $p_{\infty}=$ ambient pressure, $p_{v}=$ vapour pressure inside the bubble, $R_{0}=$ initial radius of the bubble, $b=$ initial distance from the plane wall to the centre of the bubble.

Because the flow is irrotational, the velocity vector $\mathbf{v}$ can be written in terms of a velocity potential $\phi$. Since incompressibility is assumed, $\phi$ must satisfy Laplace's equation throughout the liquid.

The pressure boundary conditions can be restated in terms of $\phi$ and $v$ with the aid of Bernoulli's equation

$$
\partial \phi / \partial t+\frac{1}{2} v^{2}+(p / \rho)=c(t) .
$$

Infinitely far from the bubble the velocity is zero, and the pressure is the ambient pressure. The velocity potential there is an arbitrary function of time only, which can be taken to be zero

$$
\operatorname{limit}_{|\mathbf{x}| \rightarrow \infty} \phi(\mathbf{x}, t)=0 \text {. }
$$

Then on the free surface,

$$
\partial \phi / \partial t+\frac{1}{2} v^{2}=\left(p_{\infty}-p_{v}\right) / \rho=\Delta p / \rho .
$$

The final boundary condition on the potential is that its normal derivative must vanish at the solid wall. Initially the potential is uniformly zero.

As a result of the assumptions, the solutions are characterized by the single parameter $b / R_{0}$. A solution for a particular value of $b / R_{0}$ can be scaled to bubbles of any initial size under any positive collapsing pressure $\Delta p$. Velocities are independent of the size of the bubble, and are scaled like $(\Delta p / \rho)^{\frac{1}{2}}$.

\section{The method of simulation}

The irrotationality of these problems is exploited by solving them in terms of the velocity potential. A number of small time steps are used. Before each step the potential problem is solved and the velocity is calculated at a large number of points representing the free surface of the cavity. The displacement of a free surface point with a velocity $\mathbf{v}$ calculated at the start of the time step is approximated by

$$
\Delta \mathbf{x}=\mathbf{v} \Delta t .
$$

The rate of change of the potential of a point moving on the free surface is

or by (3)

$$
\begin{gathered}
D \phi \mid D t=\partial \phi / \partial t+v^{2} \\
D \phi \mid D t=\Delta p / \rho+\frac{1}{2} v^{2}
\end{gathered}
$$


The change in potential of a free surface point can then be approximated by

$$
\Delta \phi=\left(\Delta p / \rho+\frac{1}{2} v^{2}\right) \Delta t
$$

A detailed description of this numerical method can be found in a recent report by Plesset \& Chapman (1970).

\section{Results of the calculations}

The collapse of an initially spherical bubble near a plane solid wall was simulated for two cases. In case 1 the parameter $b / R_{0}$ was unity; that is, the bubble boundary was in contact with the solid wall and tangent to it. In case $2 b / R_{0}$ was 1.5; the closest distance from the bubble boundary to the solid wall was initially half the radius of the bubble. Ninety-four time steps were used for case 1 and seventy-seven for case 2. Calculations were stopped when the liquid jet reached the opposite wall of the bubble since the assumption of incompressibility is no longer valid. The bubble shapes for selected time steps for cases 1 and 2 are shown superimposed in figures 1 and 2 , respectively. Table 1 lists the time intervals in units of $R_{0}(\rho / \Delta p)^{\frac{1}{2}}$ from the initiation of collapse for each shape and the downward velocity on the upper portion of the bubble at the axis of symmetry. The velocities, which are scaled like $(\Delta p / \rho)^{\frac{1}{2}}$, are given in metres/sec for the special value

$$
\frac{\Delta p}{\rho}=\frac{10^{6} \mathrm{dynes} / \mathrm{cm}^{2}}{1 \cdot 0 \mathrm{~g} / \mathrm{cm}^{3}} \approx \frac{1 \mathrm{~atm}}{\text { density of water }} .
$$

The solid wall influences the bubble early in the collapse chiefly by reducing the upward motion of the lower portion of the bubble. As a result the bubble becomes elongated in the direction normal to the wall as was predicted by Rattray (1951). The bottom of the bubble still moves upwards towards the bubble centre in case 2 , but since this upward motion is reduced, the centroid of the bubble moves towards the wall displaying the well-known Bjerknes effect.

As the bubble acquires kinetic energy, this energy is concentrated in the upper portion of the bubble which eventually flattens and forms a jet. Once the jet is formed, the speed of its tip remains fairly constant.

The behaviour of the upper portion of the bubble in case 2 is not very different from case 1. The overall shapes appear quite different, however, because the bottom of the bubble must remain in contact with the solid wall in case 1 but is allowed mobility in case 2 . The jet speed in case 2 (about $170 \mathrm{~m} / \mathrm{sec}$ under atmospheric $\Delta p$ ) is somewhat larger than the speed in case 1 (about $130 \mathrm{~m} / \mathrm{sec}$ ). This behaviour is as expected since a bubble which is farther from the wall collapses to a smaller size and can concentrate its energy over a smaller volume.

The jet appears to be the result of the deformation caused by the presence of the wall during the early part of the collapse. It is known from the linearized theory of Plesset \& Mitchell (1956) that a small deformation can lead to jetting much later in the collapse, but the jet formation found here appears before the jetting which might develop from a small initial perturbation.

Although the bubble is initially fairly close to the wall in case 2 , the final jet must pass through the liquid for a distance of more than five times its 
Vapour cavity collapse

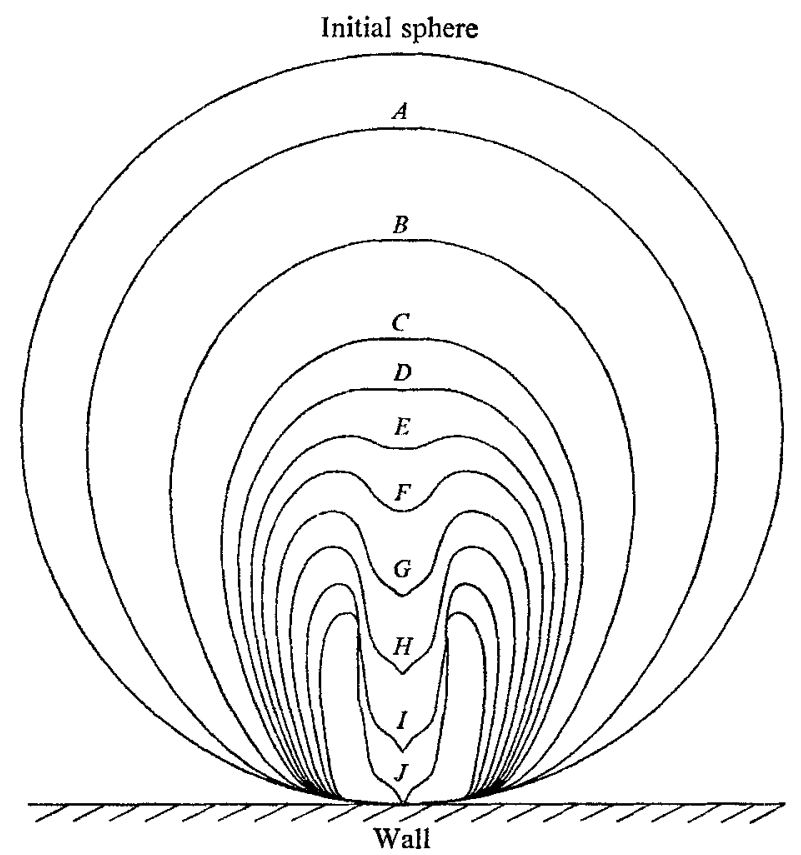

Figure 1. Bubble surfaces from case 1.
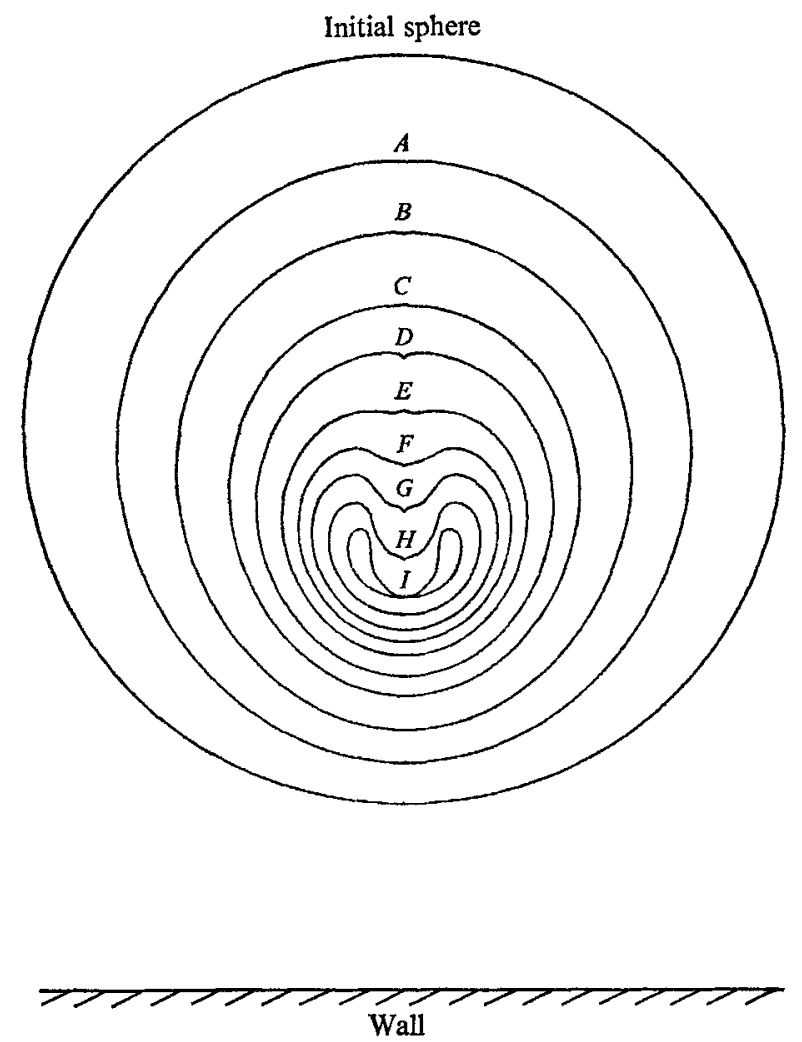

Figure 2. Bubble surfaces from case 2. 
diameter before it reaches the solid wall. The jet in case 1 , which strikes the wall directly, seems the more capable of damage even though the jet speed is lower. Apparently cavitation bubbles must almost touch the wall initially to be capable of damaging it.

\begin{tabular}{|c|c|c|c|c|}
\hline \multicolumn{3}{|c|}{ Figure 1} & \multicolumn{2}{|c|}{ Figure 2} \\
\hline Shape & $\operatorname{Tim} \theta$ & $\begin{array}{l}\text { Velocity } \\
\text { (m/sec) }\end{array}$ & Time & $\begin{array}{l}\text { Velocity } \\
\text { (m/sec) }\end{array}$ \\
\hline$A$ & $0 \cdot 63$ & $7 \cdot 7$ & 0.725 & 10 \\
\hline$B$ & 0.885 & 19 & 0.875 & 17 \\
\hline$C$ & 0.986 & 42 & 0.961 & 35 \\
\hline$D$ & 1.013 & 65 & 0.991 & 53 \\
\hline$E$ & $1 \cdot 033$ & 100 & $1 \cdot 016$ & 94 \\
\hline$F$ & $1 \cdot 048$ & 125 & 1.028 & 142 \\
\hline$G$ & 1.066 & 129 & $1 \cdot 036$ & 160 \\
\hline$H$ & 1.082 & 129 & $1 \cdot 044$ & 165 \\
\hline$I$ & 1.098 & 128 & $1 \cdot 050$ & 170 \\
\hline$J$ & $1 \cdot 119$ & 128 & - & - \\
\hline
\end{tabular}

TABLE I. Time interval from initiation of collapse, and the velocity of the bubble boundary at the axial point most distant from the wall, for the cases illustrated in figure 1 and figure 2

A jet of speed $v$ directly striking a solid boundary produces an initial pressure given by the water hammer equation,

$$
p_{W H}=\rho_{L} c_{L} v\left(\frac{\rho_{s} c_{s}}{\rho_{L} c_{L}+\rho_{s} c_{s}}\right)
$$

where the $L$ and $s$ subscripts refer to the liquid and the solid, respectively. Usually $\rho_{s} c_{s}$ is large compared to $\rho_{L} c_{L}$ producing the approximation

$$
p_{W H} \approx \rho_{L} c_{L} v .
$$

Experiments by Hancox \& Brunton (1966) have shown that multiple impacts by water at a speed of $90 \mathrm{~m} / \mathrm{sec}$ can erode even stainless steel.

Benjamin \& Ellis (1966) present two series of photographs of bubbles collapsing near a solid wall in figures 3 and 4 of their paper. The collapse illustrated in their figure 4 is very similar to case 2 . The collapse illustrated in their figure 3 falls between case 1 and case 2 . Benjamin \& Ellis estimated the jet speed in their figure 3 to be about $10 \mathrm{~m} / \mathrm{sec}$ under an ambient pressure of about $0.04 \mathrm{~atm}$. The vapour pressure of the water is very important at this reduced pressure. Since Benjamin \& Ellis did not mention the temperature of the water, this pressure cannot be determined directly. However, $\Delta p$ can be deduced from the total collapse time which they gave as $10 \mathrm{msec}$. The total collapse time for a spherical bubble is, according to Rayleigh,

$$
\tau=0.915 R_{0}(\rho / \Delta p)^{\frac{1}{2}}
$$

The total collapse times for cases 1 and 2 are only slightly greater since most 
of the time is consumed early in the collapse while the bubble is nearly spherical. For collapse near a solid wall, then, the total collapse time is roughly $\dagger$

$$
\tau \approx R_{0}(\rho / \Delta p)^{\frac{1}{2}} .
$$

Since $R_{0} \approx 1.0 \mathrm{~cm}$ and $\tau=10 \mathrm{msec}$, the pressure"difference for the collapse in figure 3 of Benjamin \& Ellis is approximately

$$
\Delta p=p_{\infty}-p_{v} \approx 10^{4} \mathrm{dynes} / \mathrm{cm}^{2} \approx 0.01 \mathrm{~atm} .
$$

A vapour pressure of $0.03 \mathrm{~atm}$ corresponds to a temperature of about $76^{\circ} \mathrm{F}$. Speeds for one atmosphere pressure difference should be increased by a factor of ten giving an estimated jet speed of roughly $100 \mathrm{~m} / \mathrm{sec}$ so that the experimental observations of Benjamin \& Ellis are compatible with the calculations performed here.

As general conclusions we may say that it appears very likely that cavitation damage with collapsing vapour bubbles is caused by the impact of the jet produced by the presence of the adjacent solid wall. Further, it appears reasonable to say that only those cavitation bubbles quite near the solid boundary can produce damage whether by a jet or by any radiated shock. From the calculations presented here, we see that for a bubble near the wall the jet is formed early in the collapse history so that the many complications of the late stages of cavity collapse do not enter. These familiar complications include the instability of the spherical shape toward the end of collapse, the effects of high bubble wall velocities on the behaviour of the vapour in the bubble, and the effects of compressibility, not only in the vapour phase, but in the liquid as well. It is also very evident that the jet appears before there is any possibility of radiating a shock.

It is not clear that the impact, or 'water-hammer' stress of $(10)$ is the mechanism of damage to the solid. For the case of the spherical bubble initially in contact with the wall and for $\Delta p=1 \mathrm{~atm}$ in water, we have $v \sim 130 \mathrm{~m} / \mathrm{sec}$ and $c_{L} \sim 1500 \mathrm{~m} / \mathrm{sec}$ so that

$$
p_{W H} \sim 2000 \mathrm{~atm} .
$$

While this is a most impressive impact stress, it is not obvious that it is the important damaging mechanism since the duration of this stress is so short. We may estimate this duration as being no longer than the time for the impact signal to traverse the radius of the jet. For a bubble with an initial radius $R_{0}=0.1 \mathrm{~cm}$, this time is $\tau_{W H} \sim 10^{-7} \mathrm{sec}$. On the other hand the stagnation pressure is approximately $\quad p_{s} \sim \frac{1}{2} \rho v^{2} \sim 800 \mathrm{~atm}$,

which will have a duration of the order of the length of the jet divided by its velocity $v$. This pressure pulse may be the source of the damage because its duration is an order of magnitude greater.

Finally, we may say that cavitation damage should have a close relationship with liquid impact damage and inferences from studies of the latter should be useful for cavitation damage. We may also use calculations of the kind presented here to get reasonably accurate estimates of cavitation stress pulses.

$\uparrow$ Rattray derived the formula

$$
\frac{\tau}{R_{0}}\left(\frac{\Delta p}{\rho}\right)^{\frac{1}{2}}=0.915\left(1+0.41 \frac{R_{0}}{2 b}\right)+O\left(\frac{R_{0}}{2 b}\right)^{2}
$$

from his perturbation analysis. 


\section{REFERENCES}

Benjamin, T. B. \& Ellis, A. T. 1966 Phil. Trans. Roy. Soc. A 260, 221.

Hancox, N. L. \& Brunton, J. H. 1966 Phil. Trans. Roy. Soc. A 260, 121.

KonNered, M. \& Stvorov, L. 1944 J. Appl. Phys. 15, 495.

Mitcheil, T. M., Krtig, C. L., Cheesewright, R. \& Hammitt, F. G. 1967 U. of Michigan, College of Eng., Rep. 07738-5-T.

Plessex, M. S. 1966 Phil. Trans. Roy. Soc. A 260, 241.

Plesset, M. S. \& Chapman, R. B. 1970 Div. of Eng. and Appl. Science, Calif. Inst. of Tech. Rep. no. 85-49.

Plesset, M. S. \& Mitchell, T. P. 1956 Quart. Appl. Math. 13, 419.

Rattray, M. 1951 Ph.D. thesis, California Institute of Technology.

Rayleigh, Lord 1917 Phil. Mag. 34, 94. 